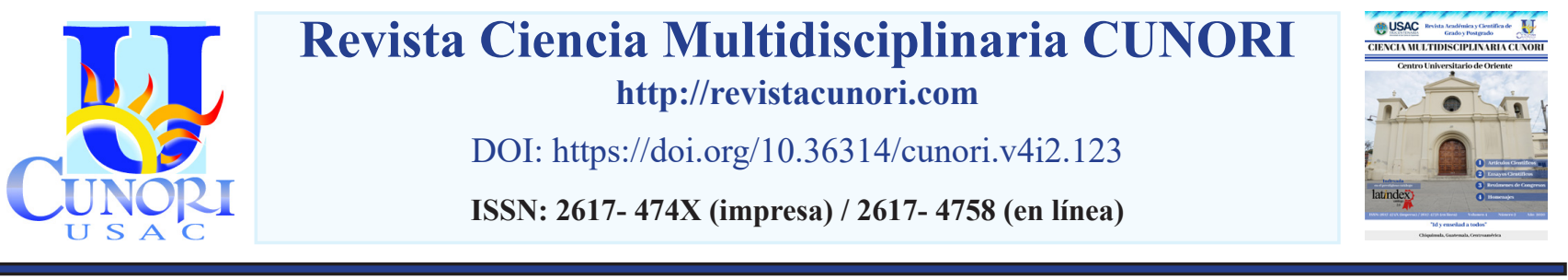

Referencia del artículo

Ordóñez-Galeano, R. (2020). Depresión y ansiedad en estudiantes de medicina. Revista Ciencia Multidisciplinaria Cunori, 4(2), 15-21. https://doi.org/10.36314/cunori.v4i2.123

\title{
Depresión y ansiedad en estudiantes de medicina
}

\section{Depression and anxiety in medical students}

\author{
Rosa Angélica Ordóñez Galeano \\ Centro Universitario de Oriente (CUNORI), Universidad de San Carlos de Guatemala \\ https://orcid.org/0000-0002-4130-7637 \\ Recibido: 30 de mayo de 2020 / Revisado: 31 de mayo de 2020 / Aceptado: 05 de junio de 2020 \\ Disponible en internet el 30 de octubre de 2020 \\ *Autor para correspondencia. \\ Correo electrónico: kikirosi02@gmail.com
}

\section{Resumen}

$\mathrm{L}$ a depresión y la ansiedad son trastornos psiquiátricos de alta prevalencia en el mundo entre el 5\% y 50\%. En el contexto del

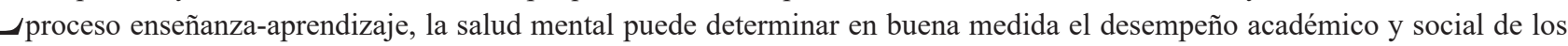
estudiantes. El objetivo es determinar la presencia de depresión y ansiedad en estudiantes de la carrera de Médico y Cirujano del Centro Universitario de Oriente. Es un estudio descriptivo transversal sobre nivel de depresión y ansiedad según Inventario de Beck, en el período de marzo-abril del año 2020. Utilizando una muestra de 359 estudiantes, recolectando la información a través de la boleta de recolección de datos autoaplicada. Se estableció predominó la ansiedad leve con un 68\% (13) y la depresión leve con un 66\%. (29). En los estudiantes que cursaban con depresión más ansiedad predominó la ansiedad severa con un 41\% (119) y la depresión moderada con un 57\% (165), encontrándose la mayoría de los estudiantes entre los 16-20 años con un 58\%. En la investigación se observó que el sexo predominante fue el femenino con 65 . El 11\% de los encuestados admitieron ser fumadores y el $31 \%$ refirió consumir con frecuencia bebidas alcohólicas. Evidenciando que el 58\% de los estudiantes repitió al menos un grado académico.

Palabras clave: ansiedad, depresión, estudiantes, inventario de beck

Abstract

$\mathrm{D}$ epression and anxiety are psychiatric disorders with a high prevalence in the world between $5 \%$ and $50 \%$. In the context of the teaching-learning process, mental health can largely determine the academic and social performance of students. The objective is to determine the presence of depression and anxiety in students of the Medical and Surgeon career at the Centro Universitario de Oriente. It is a descriptive cross-sectional study on the level of depression and anxiety according to the Beck Inventory, in the period of March-April of the year 2020. Using a sample of 359 students, collecting the information through the self-applied data collection form. Mild anxiety prevailed with 68\% (13) and mild depression with $66 \%$. (29). Severe anxiety prevailed in students with more anxiety with $41 \%$ (119) and moderate depression with 57\% (165), with the majority of students between $16-20$ years old with $58 \%$. In the research, it was observed that the predominant sex was female with $65.11 \%$ of those surveyed admitted to being smokers and 31\% reported frequently consuming alcoholic beverages. Evidence that 58\% of the students repeated at least one academic degree.

Keywords: anxiety, depression, students, Beck Inventory 


\section{Introducción}

La depresión y la ansiedad son trastornos psiquiátricos de alta prevalencia en el mundo y varían de acuerdo a las poblaciones o regiones geográficas entre 5 y 50\%. Estas condiciones representan una carga social y económica al repercutir de manera importante en la calidad de vida de las personas afectadas y en su entorno laboral y social. Debido a esto, se considera a éstas como un tema de salud pública emergente y desde hace algunos años se han empezado a generar políticas de salud para abordar dichos problemas. La ansiedad y la depresión son síndromes relacionados, considerándose que tienen un origen común y que cualquiera de ellas puede predisponer a la otra. Es importante entonces describir la presencia de ambas sintomatologías de forma individual y conjunta, y evaluar cómo se afectan entre sí (Osada et al. 2010). La depresión justifica por sí misma su estudio, ya que es uno de los problemas psicológicos que con mayor frecuencia se encuentran en la sociedad actual, a lo que no son ajenos los universitarios ya que tienen que lidiar con las exigencias sociales, familiares y con el estrés académico (Aguirre et al. 2011).

Se realizó un estudio descriptivo transversal, a través del Inventario de Beck, diagnosticando depresión y ansiedad en los estudiantes de la carrera de medicina del Centro Universitario de Oriente e identificando los factores sociodemográficos. Tomando como muestra a 359 estudiantes cursantes de primer a sexto año, durante el ciclo académico 2020. La Organización Mundial de la Salud (OMS), define a la salud mental como un estado de bienestar en el cual el individuo se da cuenta de sus propias aptitudes, puede afrontar las presiones normales de la vida, puede trabajar productiva y fructíferamente y es capaz de realizar una contribución a la comunidad. La salud mental es parte integral y esencial de la salud; de hecho, la OMS señala que no hay salud sin salud mental, refiere que los trastornos mentales representan 5 de las 10 principales cargas de morbilidad en personas de 15 a 44 años. Asimismo, de acuerdo con el proyecto Global Burden Disease de la OMS, se señala a la depresión como la principal causa de discapacidad a nivel mundial, con claro predominio en el sexo femenino y con un claro aumento en la adolescencia, siendo los trastornos más frecuentes la depresión y la ansiedad (Ortiz, 2016).

La depresión y ansiedad son problemas que trascienden socialmente, no solo por su incidencia numérica, sino también porque estos trastornos alteran en gran manera el papel bio-psico-social de quienes los experimentan (Barradas et al. 2015). El hecho de ingresar a la universidad es una fuente que puede generar experiencias de ansiedad; las clases suelen ser impartidas con metodologías diferentes, ambiente formal, siendo los exámenes el factor que genera más ansiedad en los estudiantes. En muchos casos los jóvenes universitarios provienen de distintos lugares y presentan diferencias socioeconómicas, de personalidad, de raza, credo religioso, afiliación política; lo cual podría influir. En general, diferente idiosincrasia y patrones culturales, se unen en un aula de clase para recibir una misma información y con los cuales deberán compartir muchas experiencias, lo que convierte a la vida universitaria, para muchos en atractiva, excitante y para otros, en complicada.

La salud mental en el ámbito médico se mantiene cuando los individuos pueden desempeñar de manera idónea sus capacidades personales y profesionales en condiciones adecuadas de bienestar psíquico y social. Estos requisitos cobran especial atención en el medio académico, pues éste como ninguno estimula 
la transmisión y perpetuación de actitudes, las cuales posteriormente van a tener impacto en la comunidad (Vásquez y Ríos, 2009). El Inventario de Depresión de Beck es un instrumento de autoinforme de 21 ítems diseñado para evaluar la gravedad de la sintomatología depresiva en adultos y adolescentes con una edad mínima de 13 años. En cada uno de los ítems la persona tiene que elegir, entre un conjunto de cuatro alternativas ordenadas de menor a mayor gravedad, la frase que mejor describe su estado durante las últimas dos semanas incluyendo el día en que completa el instrumento. En cuanto a su corrección, cada ítem se valora de 0 a 3 puntos en función de la alternativa escogida y, tras sumar directamente la puntuación de cada ítem, se puede obtener una puntuación total que varía de 0 a 63 . A veces, se da la circunstancia de que la persona elige más de una alternativa en un ítem dado. En este caso se toma la puntuación de la frase elegida de mayor gravedad. (Sanz J. et al 2005).

La ansiedad y la depresión se consideran dos de los desórdenes emocionales de mayor registro en los centros de salud, en población general. Así mismo dentro de la población universitaria, constituyen dos de los principales motivos de consulta atendidos a través de los servicios que presta la unidad de bienestar estudiantil universitaria (Palencia et al. 2010).

\section{Materiales y métodos}

El presente estudio se realizó en el departamento de Chiquimula con estudiantes universitarios de la carrera de médico y cirujano del Centro Universitario de Oriente, tomando en cuenta todos los grados académicos desde el 1er. hasta el 6to. grado con una muestra de 359 participantes. En el total de la población estudiada se empleó una boleta de recolección de datos, la cual se les hizo llegar por vía electrónica a los estudiantes, en la misma se encontraban interrogantes sobre los datos sociodemográficos y los correspondientes inventarios de ansiedad y depresión de Beck.

\section{Resultados}

De los 359 estudiantes incluidos en el estudio $19(5 \%)$ presentaron características clínicas de trastornos de ansiedad, 44 (12\%) presentaron depresión, 290 (81\%) estudiantes presentaron características de ansiedad más depresión y $6(2 \%)$ estudiantes no presentan características clínicas de dichos trastornos. De los 19 estudiantes con Ansiedad según el Inventario de Beck, el 68\% (13) presentaron ansiedad leve, el 11\% (2) presentaron ansiedad moderada, el 21\% (4) presentaron ansiedad severa. De los 44 estudiantes con Depresión según el Inventario de Beck, el 66\% (29) presentaron depresión leve, el 25\% (11) presentaron depresión moderada, el 9\% (4) presentaron depresión severa. De los 290 estudiantes con depresión más ansiedad, se encontró que el 28\% (81) presentó depresión leve y un 29\% (84) ansiedad leve, un 57\% (165) presentó depresión moderada y un 30\% (87) ansiedad moderada, un 15\% (44) presentó depresión severa y un 41\% (119) ansiedad severa. Se observó que el sexo predominante fue el femenino con 65 . El 11\% de los encuestados admitieron ser fumadores y el 31\% refirió consumir con frecuencia bebidas alcohólicas. Evidenciando que el $58 \%$ de los estudiantes repitió al menos un grado académico. 
Figura 1. Distribución de depresión y/o ansiedad según Inventarios de Beck en estudiantes de la Carrera de Medicina del Centro Universitario de Oriente CUNORI, en el período de marzo-abril de 2020.

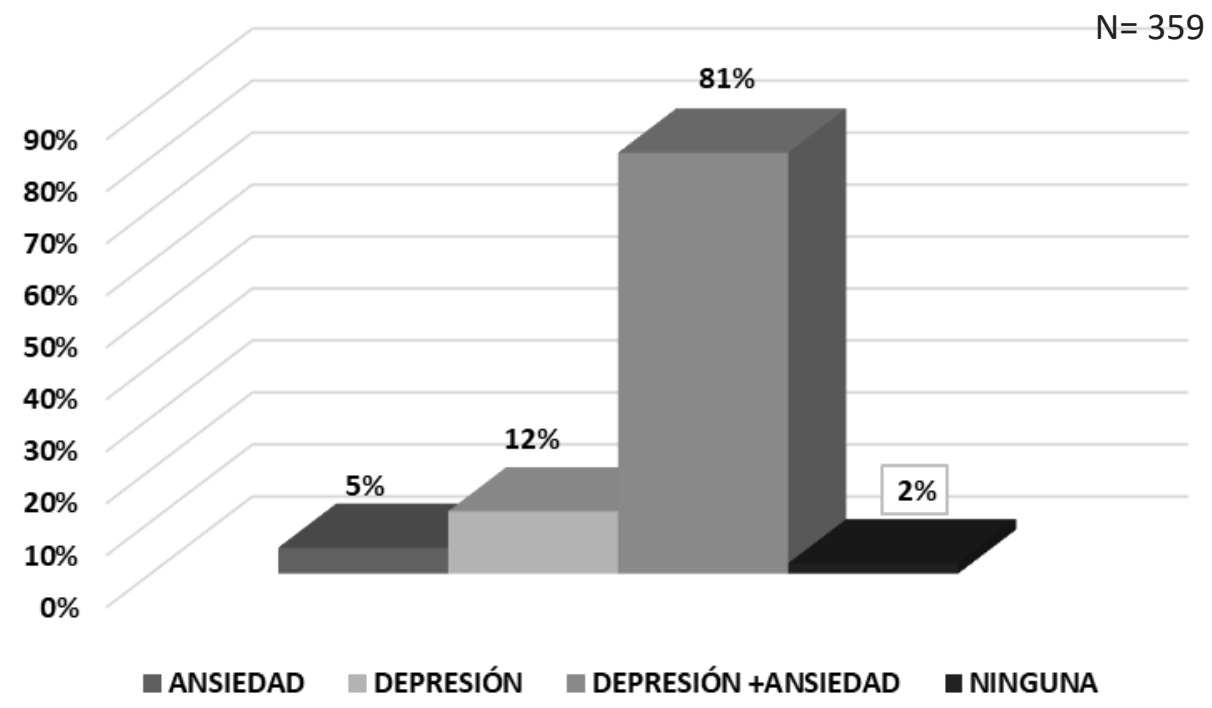

Ordóñez-Galeano (2020). Base de datos del estudio de campo.

Como se observa en la figura 19 Estudiantes $(5 \%)$ presentaron características clínicas de trastornos de ansiedad, 44 (12\%) presentaron depresión, 290 (81\%) estudiantes presentaron características de ansiedad más depresión y $6(2 \%)$ estudiantes no presentaron ninguna de estas patologías.

Figura 2. Distribución Distribución según nivel de ansiedad en base al inventario de Beck, en estudiantes de la Carrera de Medicina del Centro Universitario de Oriente CUNORI, en el período de marzo - abril de 2020.

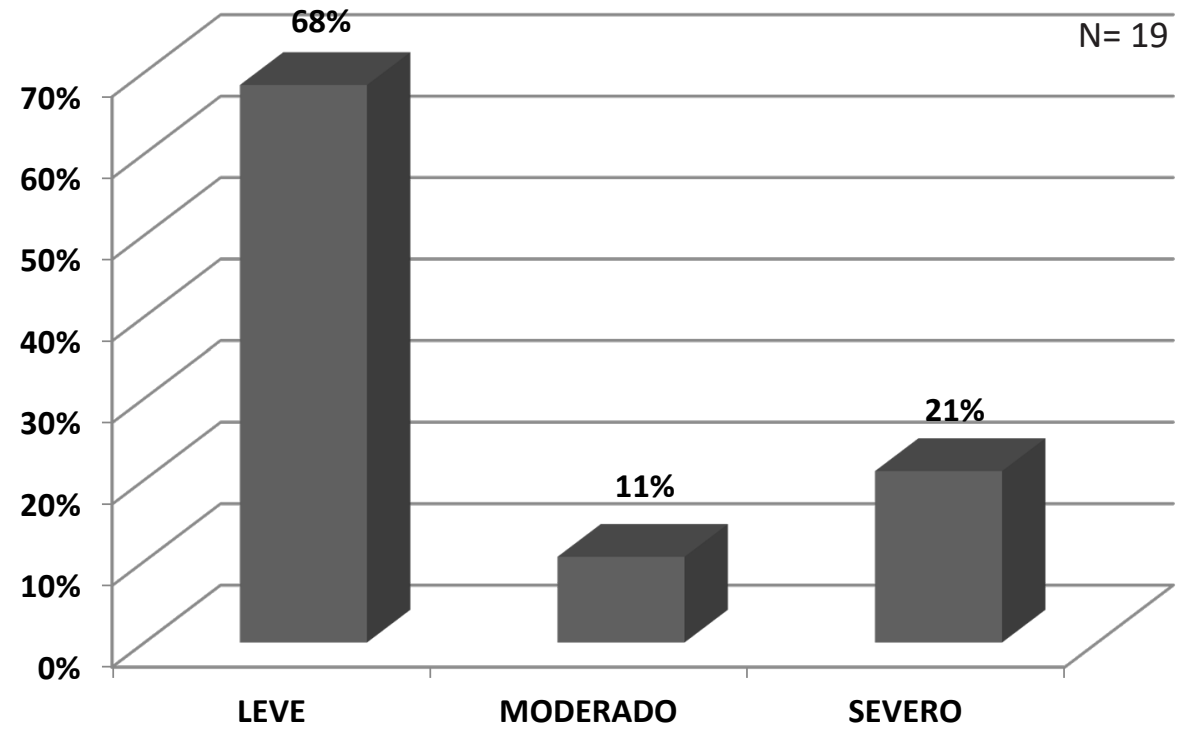

Ordóñez-Galeano (2020). Base de datos del estudio de campo. 
El 68\% (13) de los estudiantes presentaron ansiedad leve, el 11\% (2) presentaron ansiedad moderada, el $21 \%$ (4) presentaron ansiedad severa.

Figura 3. Distribución Distribución según nivel de depresión en base al inventario de Beck, en estudiantes de la Carrera de Medicina del Centro Universitario de Oriente CUNORI, en el período de marzo - abril de 2020.

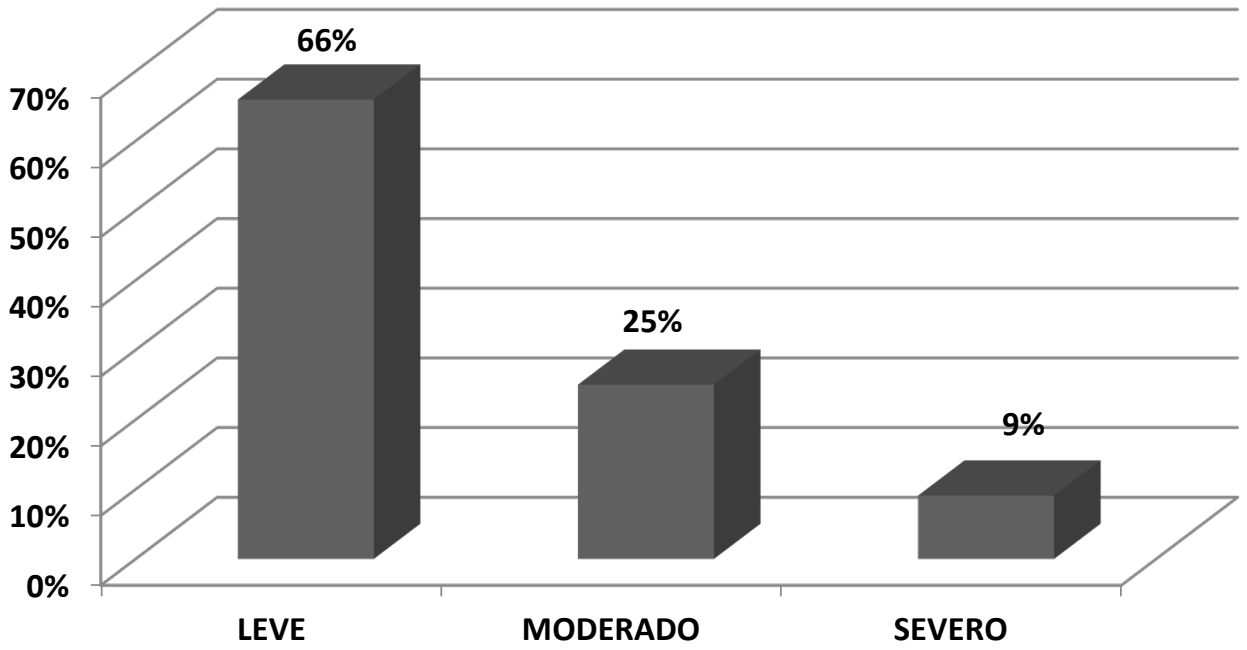

Ordóñez-Galeano (2020). Base de datos del estudio de campo.

El 66\% (29) de los estudiantes presentaron depresión leve, el 25\% (11) presentaron depresión moderada, el 9\% (4) presentaron depresión severa.

Figura 4. Distribución según nivel de Depresión más Ansiedad en base al inventario de Beck, en estudiantes de la Carrera de Medicina del Centro Universitario de Oriente CUNORI, en el período de marzo - abril de 2020

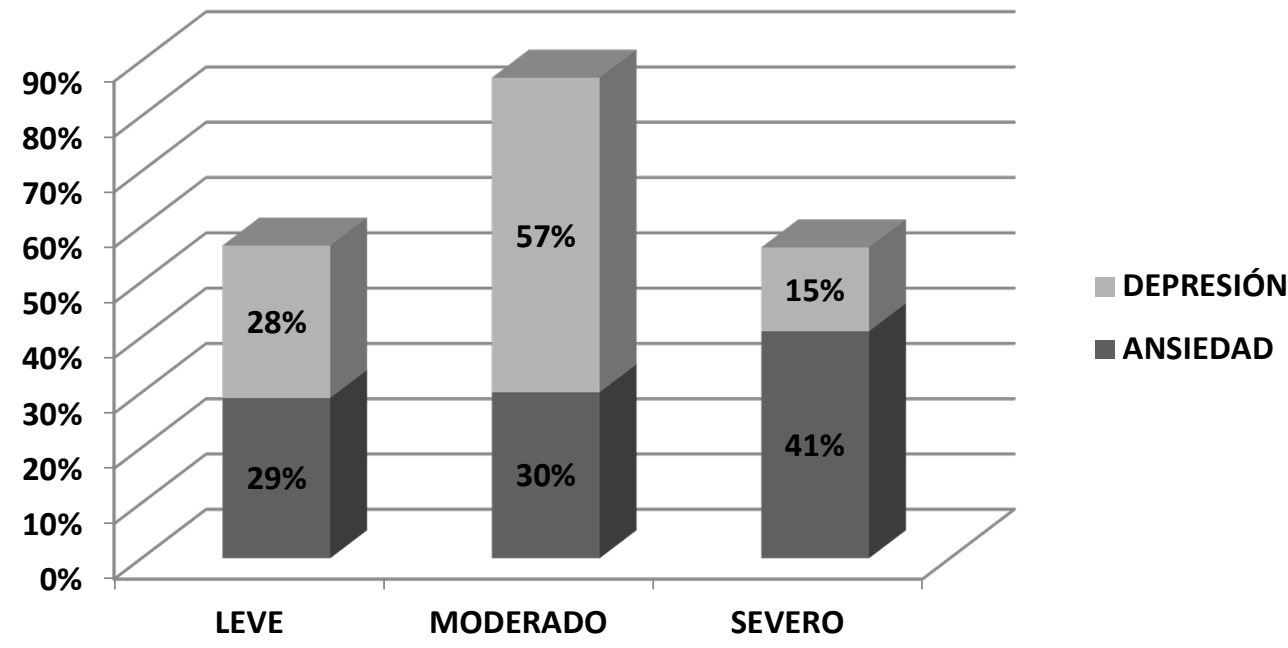

Ordóñez-Galeano (2020). Base de datos del estudio de campo. 
De los 290 estudiantes con depresión más ansiedad, se encontró que el 28\% (81) presentó depresión leve y un 29\% (84) ansiedad leve, un 57\% (165) presentó depresión moderada y un 30\% (87) ansiedad moderada, un 15\% (44) presentó depresión severa y un 41\% (119) ansiedad severa.

\section{Discusión}

Se determinó que la prevalencia de ansiedad y/o depresión según Inventario de Beck fue de 98\% en los estudiantes. Estableciendo que predominó la ansiedad leve con un 68\% (13) y la depresión leve con un 66\% (29). En los estudiantes que cursaban con depresión más ansiedad predominó la ansiedad severa con un 41\% (119) y la depresión moderada con un 57\% (165). Estando estos datos por encima de las cifras que maneja la población en general, tomando en cuenta que la población estudiantil, y específicamente los estudiantes de medicina se encuentran más vulnerables frente a los diferentes escenarios que les plantean los estudios superiores; considerando también aspectos familiares, psicosociales y la exigencia académica.

En la investigación se observó que la mayoría de los estudiantes se encontraba entre los 16-20 años de edad con un 58\% (204). El sexo predominante fue el femenino con 65\% (229), lo que coincide con los indicadores de "feminización" de la población universitaria, especialmente en la carrera de medicina, de manera que es lógico suponer que este aumento en número de estudiantes mujeres conlleva a un aumento en la población estudiantil de los trastornos que más prevalecen en este sexo.

El 11\% de los encuestados refirieron ser fumadores y el 31\% refirió consumir con frecuencia bebidas alcohólicas, ya que muchos estudiantes practican el consumo de estas sustancias que pueden dañar su salud, para afrontar las situaciones estresantes que implica su labor académica. También se evidenció que el 58\% (204) de los estudiantes repitió al menos un grado académico, tomando en cuenta que el padecimiento de algún tipo de estos trastornos puede influir en el rendimiento académico. Todos estos datos brindan información importante, la cual permite tener un conocimiento de la situación de salud mental de la población estudiantil, la misma que servirá para gestionar protocolos de atención temprana en psiquiatría y psicología, en el área de bienestar estudiantil.

\section{Agradecimientos}

Al coordinador de la carrera y destacado catedrático, Dr. Ronaldo Armando Retana Albanés por autorizar la realización del estudio. Al Comité Organizador de Trabajos de Graduación de Medicina por aprobar el tema a investigar. Al revisor Dr. Carlos Iván Arriola Monasterio, a la asesora, Dra. Edna Anabella Julián Leal. Al Centro Universitario de Oriente de la Universidad de San Carlos de Guatemala. 


\section{Referencias}

Barradas Alarcón, M.; Fernández Mojica, N.; Robledo Salinas, M.; Delgadillo Castillo, R. y Luna Martínez, M. (2015). Trastornos afectivos: nivel de ansiedad y depresión en universitarios. Revista Iberoamericana de Producción Académica y Gestión Educativa 2(3):1-17. http://www.pag.org.mx/ index.php/PAG/article/view/493/532

Ortiz Castillo, J. (2016). Relación de la ansiedad y depresión sobre el rendimiento académico en estudiantes de medicina. Revista Psiquiatría y Salud Mental 3(4):123-131. http://www.schilesaludmental.cl/pdf/REVISTA\%203-4-2016/(5)\%20Salud\%20 Mental,\%20ansiedad,\%20depresión,\%20 rendimiento $\% 20$ académico, $\% 20$ Est.\%20medicina $\% 20123-131$.pdf

Osada, J.; Rojas, M.; Rosales, C. y Vega-Dienstmaier, J. (2010). Sintomatología ansiosa y depresiva en estudiantes de medicina. Revista de Neuro-Psiquiatría 73(1):15-19. DOI:https://doi.org/10.20453/ rnp.v73i1.1651

Palencia, F.; Peñate Rodríguez, F. y Rosado Castillo, E. (2010). Sintomatología de ansiedad y depresión en estudiantes de medicina (Tesis Licenciatura). Guatemala, USAC, Facultad de Ciencias Médicas. 65 p. http://bibliomed.usac.edu.gt/tesis/pre/2010/042.pdf

Sanz, J.; García-Vera, M.; Espinosa, R.; Fortún, M. y Vázquez, C. (2005). Adaptación española del Inventario para la Depresión de Beck-II (BDI-II): 3; propiedades psicométricas en pacientes con trastornos psicológicos (en línea). Revista Clínica y Salud 16(2):121-142. https://www.redalyc.org/ pdf/1806/180616104001.pdf

Vásquez Ventura, C. y Ríos Morales, J. (2009). Prevalencia de estrés, ansiedad y depresión en estudiantes de postgrado. Revista Neurología, Neurociencia y Psiquiatría 42(1-4):7-12. https://www.medigraphic.com/cgi bin/new/resumen.cgi?IDARTICULO=70707

\section{Sobre la autora}

\section{Rosa Angélica Ordóñez Galeano}

Médica y Cirujana egresada del Centro universitario de Oriente de la Universidad de San Carlos de Guatemala.

Copyright (c) Rosa Angélica Ordóñez Galeano

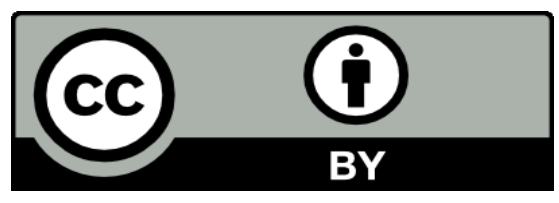

Este texto está protegido por una licencia CreativeCommons 4.0.

Usted es libre para compartir, copiar y redistribuir el material en cualquier medio o formato y adaptar el documento, remezclar, transformar y crear a partir del material para cualquier propósito, incluso comercialmente, siempre que cumpla la condición de atribución: usted debe reconocer el crédito de una obra de manera adecuada, proporcionar un enlace a la licencia, e indicar si se han realizado cambios. Puede hacerlo en cualquier forma razonable, pero no de forma tal que sugiera que tiene el apoyo del licenciante o lo recibe por el uso que hace. 